\title{
CHAOTIC LAYERS IN RESONANCE PROBLEMS
}

\author{
JACQUES HENRARD, MICHÈLE MOONS and ALESSANDRO MORBIDELLI \\ Département de mathématique FUNDP \\ 8, Rempart de la Vierge, B-5000 Namur, Belgique
}

\begin{abstract}
The recent numerical simulations of Tittemore and Wisdom $(1988,1989,1990)$ and Dermott et al. (1988), Malhotra and Dermott (1990) concerning the tidal evolution through resonances of some pairs of Uranian satellites have revealed interesting dynamical phenomena related to the interactions between close-by resonances. These interactions produce chaotic layers and strong secondary resonances. The slow evolution of the satellite orbits in this dynamical lanscape is responsible for temporary capture into resonance, enhancement of eccentricity or inclination and subsequent escape from resonance. The present contribution aims at developing analytical tools for predicting the location and size of chaotic layers and secondary resonances. The problem of the 1:3 inclination resonance between Miranda and Umbriel is analysed.
\end{abstract}

Key words: orbit-orbit resonance - chaotic layers - secondary resonance - perturbation theory.

\section{Introduction}

Dissipation of energy in the tides raised by satellites on the planet causes the orbits of satellites to expand at different rates, thus changing the ratios of their mean motions. Occasionally the mean motions of two of them approach a low order $p: q$ commensurability (with $p, q$ small integers). Goldreich (1965) has shown that such a commensurability can be maintained during further tidal evolution of the satellites orbits, thus providing an explanation of the frequent occurence of such commensurabilities in the Solar System.

The mechanism is as follows. The perturbing potential of the two satellite interaction contains the following angular variables:

$$
\theta_{i, j, k, \ell}=p \lambda^{\prime}-q \lambda+i \Omega^{\prime}+j \Omega+k \omega^{\prime}+\ell \omega,
$$

where $\lambda^{\prime}, \lambda$ are the mean longitudes, $\boldsymbol{\Omega}^{\prime}, \boldsymbol{\Omega}$ the longitudes of the ascending nodes and $\omega^{\prime}, \omega$ the arguments of pericenter of the two satellites. Each of these angular variables can excite a resonance, i.e. can enter into libration about some constant value.

In the single resonance approximation used in the studies of resonances amongst the Jovian and Saturnian satellites (e.g. Goldreich 1965, Yoder 1973, Peale 1986) only one of these angular variables is retained in the analysis. Indeed, due to the differential precession of nodes and pericenters imposed by the oblateness of the planet, when one of these angular variables is very slow the other ones are not. Therefore, they can be averaged out of the potential. This single resonance approximation lends itself to a simple and almost intuitive treatment which allows an analytical derivation of the conditions for - and the probability of - capture into resonance and also describes the subsequent orbital evolution (Yoder 1979, Henrard 1982, Henrard and Lemaître 1983).

In the case of the satellites of Uranus this single resonance approximation may not be good enough even for a purely qualitative analysis. Indeed, as pointed out by Dermott (1984), the oblateness of Uranus is significantly smaller than the oblateness of Jupiter and Saturn and thus the differential precession of nodes and pericenters 
does not separate very well the various resonances. This was not analysed further for lack of motivation because the satellites of Uranus are not presently in resonance.

But, more recently, it has been suggested by Peale (1988) that temporary capture into resonance could have played a role in the history of these satellites. The unexpected thermal history of some of them, revealed by the cameras of Voyager II, could have been caused by a temporary increase in their eccentricity due to a temporary capture into resonance. Also the anomalous high inclination of Miranda could be a remnant of such a temporary capture.

The numerical experimentations by Tittemore and Wisdom $(1988,1989,1990)$ and by Dermott et al. (1988) and Malhotra and Dermott (1990) have shown indeed that temporary capture in resonance is a likely outcome of the tidal expansion of the orbits in the case of the satellites of Uranus. For the 1:3 resonance in inclination of Miranda and Umbriel (which has been the most thoroughly investigated) the mechanism is as follows: first Miranda is captured into the so called $i_{M}^{2}$-resonance with a small inclination. At this stage the single resonance approximation is still valid. Later on, as the inclination of Miranda grows as predicted by the single resonance theory, the interaction between the $i_{M}^{2}$-resonance and the other nearby resonances produces secondary commensurabilities between the libration frequency and the other slow frequencies. This is a new phenomenon which does not appear in the resonances amongst the satellites of Jupiter and Saturn (but does appear in the asteroidal resonances as pointed out by Henrard and Lemaître 1986). If Miranda is captured into one of these secondary resonances, it is dragged into the chaotic layer which marks the boundary of the primary $i_{M}^{2}$-resonance, whereupon it can escape from the commensurability.

The main features of the phase space which permit such a scenario are the secondary resonances and the chaotic layer bordering the primary resonance. We shall summarize in this report our results concerning the description of such features. A first order analysis with the semi-numerical method described in (Henrard 1990) gives a first overall view of the dynamical landscape. The location of secondary resonances and chaotic layers are obtained with good accuracy but the size of the chaotic layers is systematically underestimated. More details about this analysis are given in (Moons and Henrard 1992). A more refined analysis based upon the technique of successive eliminations developed in (Morbidelli 1992) shows that actually the size of the chaotic layers is enhanced by the overlapping of secondary resonances that a first order theory does not reveal.

\section{Modelization of the 1:3 Miranda-Umbriel inclination resonance}

We modelize the interaction Umbriel-Miranda by the Hamiltonian function:

$$
\begin{aligned}
H= & C(N-S)^{2}+A N+2 D S \cos 2 \sigma \\
& +d_{1} i_{U} \sqrt{2 S} \cos (\sigma-\nu)+d_{2} i_{U} \sqrt{2 S} \cos (\sigma+\nu)+d_{3} i_{U}^{2} \cos 2 \nu,
\end{aligned}
$$


where

$$
\begin{aligned}
A & =5.93910^{-4}, \quad C=-1.62278, \\
D & =-1.73210^{-6}, \quad d_{1}=2.88410^{-6} \\
d_{2} & =-4.95310^{-6}, \quad d_{3}=-1.20010^{-6}
\end{aligned} .
$$

The angular variables are the usual resonance variables:

$$
\sigma=\left(-\lambda_{M}+3 \lambda_{U}-2 \Omega_{M}\right) / 2 \quad, \quad \nu=\left(\lambda_{M}-3 \lambda_{U}+2 \Omega_{U}\right) / 2
$$

where $\lambda$ is the mean longitude, $\Omega$ the longitude of the node and $i$ the inclination of respectively Miranda (when the subscript is $M$ ) or Umbriel (when the subscript is $U)$. The momenta conjugated to them are:

$$
S=\left(1-\cos i_{M}\right) \sqrt{(1-\mu) a_{M}} \quad, \quad N=2 \sqrt{(1-\mu)}\left(\sqrt{a_{M}}-\sqrt{a^{\star}}\right)+S .
$$

where $a^{\star}=0.48071$ is the "exact resonance" value of the semi-major axis of Miranda.

This Hamiltonian based upon the restricted problem of three bodies includes the effects of the oblateness of Uranus and of the satellite-satellite interaction (up to degree 2 in the inclinations) and is averaged over the orbital frequencies, retaining only the secular terms and the long period terms due to the 1:3 commensurability between the orbital frequencies. Both satellites are assumed to be on a circular orbit (see Moons and Henrard 1992).

At the zeroth order approximation (when $D=d_{1}=d_{2}=d_{3}=0$ ) the frequencies of the periodic terms of (2) are:

$$
\begin{gathered}
{\left[\frac{d}{d t}(-2 \nu)\right]_{0}=-4 C(N-S)-2 A,} \\
{\left[\frac{d}{d t}(\sigma-\nu)\right]_{0}=-4 C(N-S)-A,} \\
{\left[\frac{d}{d t}(2 \sigma)\right]_{0}=-4 C(N-S),} \\
{\left[\frac{d}{d t}(\sigma+\nu)\right]_{0}=A .}
\end{gathered}
$$

When the oblateness of the planet (i.e. the coefficient $A$ ) is large enough, these frequencies are well separated and when one of them is small the other ones are large enough. The corresponding periodic terms can be averaged out. For instance when the frequency of $\sigma$ is close to zero (i.e. when $N-S$ is close to zero) the Hamiltonian (2) can be simplified to:

$$
H_{0}=C(N-S)^{2}+A N+2 D S \cos 2 \sigma .
$$

which is integrable. The momentum $N$ is a constant and the phase space of the $(\sigma, S)$ degree of freedom is shown in cartesian coordinates $y=\sqrt{2 S} \sin \sigma, x=\sqrt{2 S} \cos \sigma$ in Figure 1. For values of $N$ larger than $-D / C$, the phase space shows a zone 

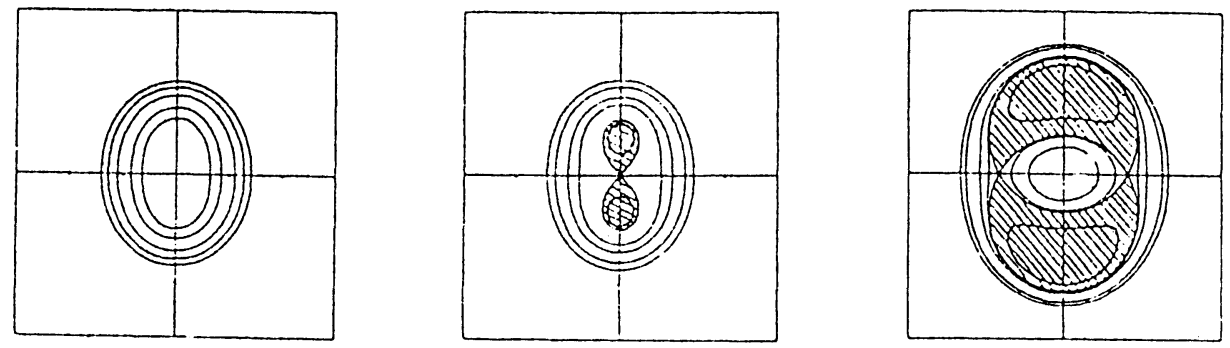

Fig. 1. Typical phase space portraits of the Hamiltonian $H_{0}$ in the plane $(x, y)$. The first figure is for $N<-D / C$, the second one for $-D / C<N<D / C$ and the third one for $N>D / C$. The resonance zone is shaded.

in which the angle $\sigma$ librates (shaded in Figure 1). We shall call such a zone the resonance zone.

For smaller values of the oblateness of the planet (i.e. when $A$ is smaller) we can no longer neglect the other periodic terms. The Hamiltonian $H_{0}$ can still be taken as a first approximation of the system but the remaining terms,

$$
H_{1}=d_{1} i_{U} \sqrt{2 S} \cos (\sigma-\nu)+d_{2} i_{U} \sqrt{2 S} \cos (\sigma+\nu)+d_{3} i_{U}^{2} \cos 2 \nu
$$

have to be taken into account as they play an essential role as seen in the following section.

\section{Surfaces of section}

This section is devoted to an analytical study of the Hamiltonian (2). This Hamiltonian is composed of two parts: $H_{0}$, a two-degree of freedom separable Hamiltonian (see 7), and $H_{1}$, a perturbation which is proportional to the inclination of Umbriel (see 8).

First of all, we shall introduce action-angle variables for $H_{0}(\sigma, S, N)$ and compute the unperturbed frequencies in order to locate the secondary resonances at work. After that, we shall take into account the perturbation $H_{1}(\sigma, S, \nu, N)$ and, applying a semi-numerical first order perturbation method (Henrard 1990), we shall draw the level curves of a quasi-integral of motion on surfaces of section in order to determine the regular and the chaotic zones.

When comparing these results with those of the numerical integration, we shall see that the semi-numerical method accurately predicts the location and the shape of the secondary resonances as well as the location of the chaotic layers; the size of the latest, however, is systematically underestimated. 


\subsection{ACtion-ANGLe Variables}

The action-angle variables for the Hamiltonian $H_{0}$ are defined by

$$
\begin{array}{ll}
\psi_{1}=\frac{2 \pi}{T_{1}} t & , \quad J_{1}=\frac{1}{2 \pi} \oint S d \sigma \\
\psi_{2}=\nu-\rho\left(t, H_{0}, N\right) & , \quad J_{2}=N
\end{array}
$$

where $t\left(\sigma, H_{0}, N\right)$ denotes the time along the periodic trajectories (starting at $\sigma=$ $\frac{\pi}{2}$ ) of the unperturbed Hamiltonian $H_{0}$ considered as a one-degree of freedom Hamiltonian in $(\sigma, S)$ depending on a parameter $N . T_{1}$ denotes the period of the trajectories and $\rho\left(t, H_{0}, N\right)$ is a $T_{1}$-periodic function in $t$ :

$$
\rho=\int_{0}^{t}\left[\frac{\partial H_{0}}{\partial N}-\left\langle\frac{\partial H_{0}}{\partial N}\right\rangle\right] d t^{\prime}
$$

The phase space of this Hamiltonian has already been shown in Figure 1 in the previous section.

The actions $J_{i}$ are constant along the periodic trajectories of $H_{0}\left(2 \pi J_{1}\right.$ is the area of the trajectory) while the angles $\psi_{i}$ are linear functions of the time.

\subsection{Frequencies aNd SECONDARY RESONANCES}

When expressed in $\left(\psi_{i}, J_{i}\right)$, the Hamiltonian $H_{0}$ is transformed into $K_{0}\left(J_{1}, J_{2}\right)$. The explicit form of $K_{0}$, however, is not known, but the frequencies

$$
\begin{aligned}
& \omega_{1}=\dot{\psi}_{1}=\frac{\partial K_{0}}{\partial J_{1}}=\frac{2 \pi}{T_{1}} \\
& \omega_{2}=\dot{\psi}_{2}=\frac{\partial K_{0}}{\partial J_{2}}=\frac{1}{T_{1}} \oint \frac{\partial H_{0}}{\partial N} d t
\end{aligned}
$$

as well as the value of the action-angle variables at any time (at this stage, we don't need it) can be computed by numerically integrating the canonical equations associated to (7).

Taking a set of initial conditions in semi-major axis and inclination of Miranda, or equivalently (see 5) in $S$ and $N$, we can compute the corresponding values of $\omega_{1}$ and $\omega_{2}$ and, finally, plot the level curves of $\omega_{2} / \omega_{1}$, which gives the location of the secondary resonances (see Figure 2).

Let us remark that the transformation (9) cannot be defined for the set of initial conditions corresponding to the separatrix of the first degree of freedom, as the period becomes infinite. There is a discontinuity in the definition of the actionangle variables when crossing the separatrix, but the transformation (9) and the frequencies (11) can be computed numerically with high accuracy if we except the separatrix and a small neighbourhood of it. 


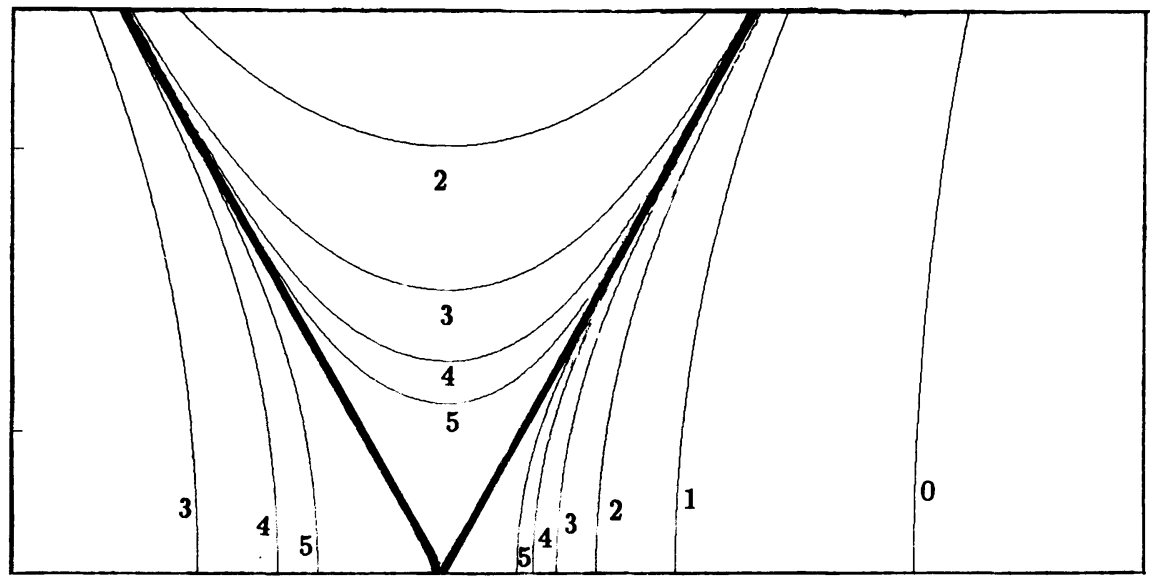

Fig. 2. Location of the secondary resonances in the plane $\left(a_{M}, i_{M}\right)$. On the horizontal axis, we have $0.4806 \leq a_{M} \leq 0.4809$ while, on the vertical axis, we have $0 . \leq i_{M} \leq 0.1$. The $N / 1$ secondary resonances $\left(\omega_{2} / \omega_{1}=N\right)$ are labelled $N$. The thick V-shaped line is the separatrix of the first degree of freedom.

\subsection{Perturbation method}

The transformation (9) reduces the Hamiltonian $H=H_{0}+H_{1}$ to

$$
K\left(\psi_{1}, \psi_{2}, J_{1}, J_{2}\right)=K_{0}\left(J_{1}, J_{2}\right)+K_{1}\left(\psi_{1}, \psi_{2}, J_{1}, J_{2}\right)
$$

with $K_{1}$ of the order of $i_{U}$ with respect to $K_{0}$ and we may apply a perturbation method to eliminate the angular dependance. While doing so, we have two problems:

1. the form (12) is not explicitely known (this is not really a problem).

2. we have to take care of the secondary resonances

The semi-numerical first order perturbation method (Henrard 1990) allows us to compute the result of the elimination of all but one (the resonant one) angular combinations. Practically, we have to split up the phase space in zones, each containing only one secondary resonance $\frac{\omega_{2}}{\omega_{1}}=j$. For each zone we introduce the unimodular transformation

$$
\begin{array}{lll}
\theta_{1}=j \psi_{1}-\psi_{2} & , & I_{1}=-J_{2} \\
\theta_{2}=\psi_{1} & , & I_{2}=J_{1}+j J_{2}
\end{array}
$$

to get new action-angle variables appropriate to the secondary resonance involved.

With the help of the semi-numerical method, we can compute the average of $K$ with respect to $\theta_{2}$ (Henrard 1990, Henrard and Moons 1992). The result we are interested in here, however, is the value of the quasi-integral of motion

$$
\bar{I}_{2}=J_{1}+j J_{2}+\frac{\partial W}{\partial \theta_{2}}
$$

and the way of computing the value of this quasi-integral on surfaces of section defined by $\psi_{1}=0$ (or, equivalently, $\sigma=\frac{\pi}{2}$ ) and $H=h$ is also explained in (Henrard 1990, Moons and Henrard 1992). 


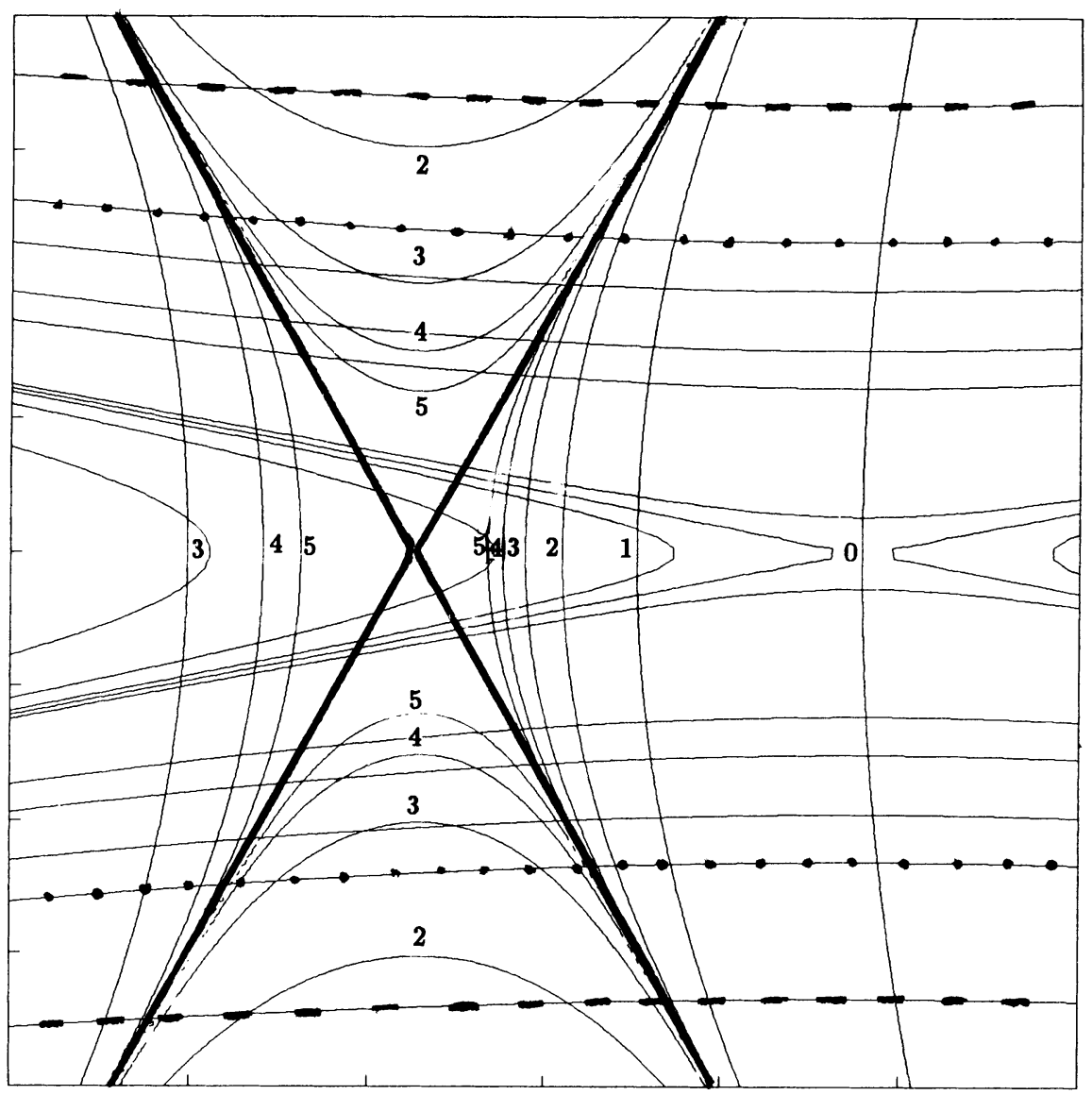

Fig. 3. Trace of the surfaces $\left(\sigma=\frac{\pi}{2}, H=h\right)$ and of the secondary resonances $\left(\omega_{2} / \omega_{1}=N\right)$ in the plane $\left(a_{M},-i_{M} \sin \nu\right)$ for $0.4806 \leq a_{M} \leq 0.4809$ and $-0.1 \leq-i_{M} \sin \nu \leq 0.1$. The $N / 1$ secondary resonances are labelled $N$. The dashed line corresponds to $h=1.510^{-6}$ and the dotted line to $h=7.510^{-7}$.

\subsection{SuRfaCES OF SECTION}

The surfaces of section $\left(\sigma=\frac{\pi}{2}, H=h\right.$ ) are hyperboloïd-like surfaces (of one or two sheets according to the value of $h)$ in the three-dimensional space $\left(a_{M},-i_{M} \sin \nu\right.$, $\left.i_{M} \cos \nu\right)$.

We have plotted in Figure 3 the trace of these surfaces in the plane $\left(a_{M},-i_{M} \sin \nu\right)$ as well as the trace of the secondary resonances.

We present here the results on surfaces corresponding to the values $h=1.510^{-6}$ and $h=7.510^{-7}$; other results can be found in (Moons and Henrard 1992).

As we see in figure 3 , the surface corresponding to $h=1.510^{-6}$ crosses the $0 / 1$ and the $1 / 1$ secondary resonances in the internal zone (zone of clockwise circulation for the angle $\sigma$ ), crosses twice the $2 / 1$ secondary resonance in the resonance zone and 


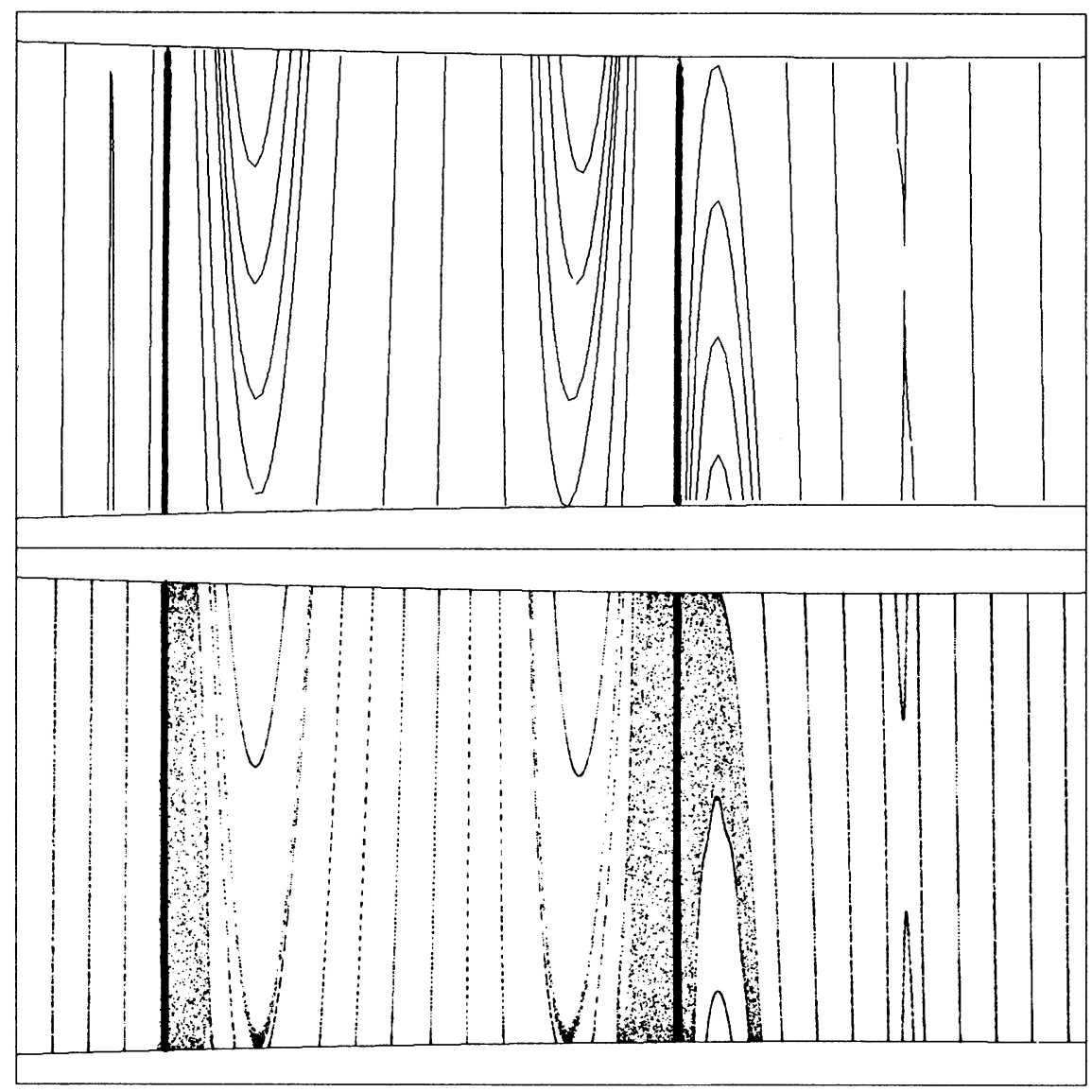

Fig. 4. Plot of the projection on the plane $\left(a_{M},-i_{M} \sin \nu\right)$ of the quasi-integral $I_{2}$ (top panel) and of the solution of the differential equations (bottom panel) evaluated for $\left(\sigma=\frac{\pi}{2}\right.$, $\left.h=1.510^{\circ}\right)$. For each panel, we have, on the horizontal axis, $0.4806 \leq a_{M} \leq 0.4809$ and, on the vertical axis $-0.1 \leq-i_{M} \sin \nu \leq 0.1$. The thick line is the separatrix of the first degree of freedom. From the left to the right, we see: the $3 / 1$ resonance in the external zone, the $2 / 1$ resonance in the resonance zone, the $1 / 1$ and the $0 / 1$ resonances in the internal zone.

crosses the $3 / 1$ secondary resonance in the external zone (zone of counterclockwise circulation for the angle $\sigma$ ). The secondary resonances encountered for $h=7.510^{-7}$ are a little bit different: the $2 / 1$ secondary resonance is now appearing (for $h=$ $1.510^{-6}$ it was embedded in the chaotic zone) and the secondary resonance we find now in the resonance zone is the $3 / 1$ resonance.

Let us now draw the level curves of the quasi-integral $\bar{I}_{2}$ (see 14) on those surfaces of section and compare the results with the numerical integration of the differential equations associated to $H$ (see 2).

The results are presented in Figure 4 (for $h=1.510^{-6}$ ) and figure 5 (for 


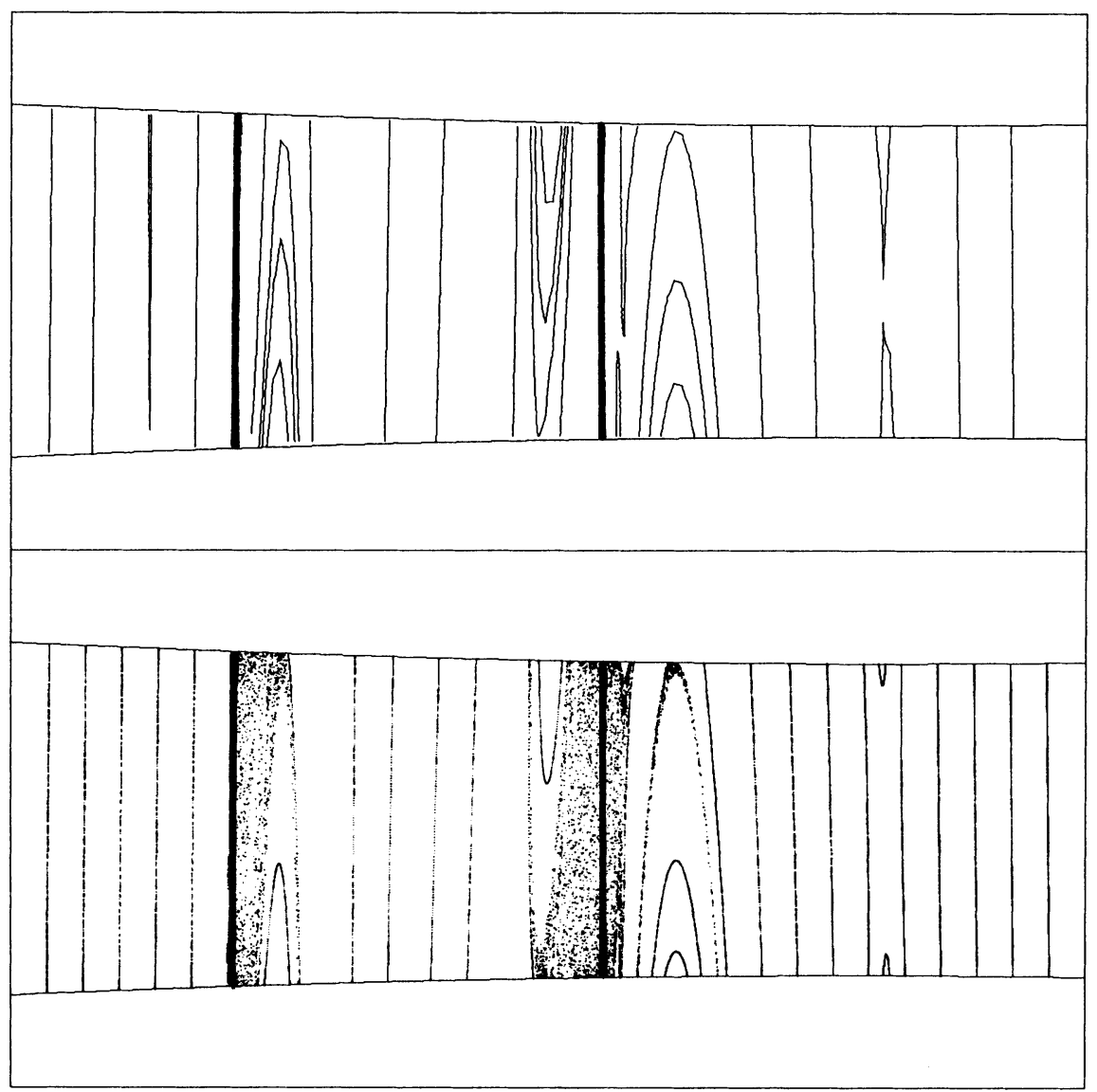

Fig. 5. Same as figure 4 , but for $h=7.510^{-7}$. The resonances appearing here are, from the left to the right: the $3 / 1$ resonance in the external zone, the $3 / 1$ resonance in the resonance zone, the $2 / 1,1 / 1$ and $0 / 1$ resonances in the internal zone.

$h=7.510^{-7}$ ) which are composed of two panels: the top one is the result of the semi-numerical method while the bottom one is the result of the numerical integration. In those figures, we have represented only one half of the hyperboloîd surface by projection on the plane $\left(a_{M},-i_{M} \sin \nu\right)$; due to the symmetry of the problem, however, the other half is identical to it.

As we can see, there is a very good agreement between the top and the bottom panels with respect to the shape and the location of the secondary resonances. However, there is a problem concerning the size of the chaotic zone: in the top panel of figure 4 , we remark that the $1 / 1$ resonance seems well separated from the separatrix while, in the bottom panel,we see that it is included in the chaotic zone. The same phenomenon appears, but in a reduced manner, in figure 5 with the $3 / 1$ resonance (in the resonance zone) and the $2 / 1$ resonance (in the internal zone). 
This "underestimation" of the size of the chaotic zone is due to the fact that the semi-numerical method is only a first order method and, hence, is not appropriate for zones where resonances overlap; we shall see in the next section a more accurate (but more sophisticated) way to do it. Anyway, the method used here is very simple and very useful to locate and characterize roughly the secondary resonances.

\section{Successive eliminations of perturbation harmonics}

The results illustrated in section 2, show the power of the action-angle variable semi-numerical perturbation method developed by J. Henrard (1990). However some limits are, nevertheless, evident. First of all such a method, the advantage of which is to take completely into account the dynamical effect of the most relevant degree of freedom $\left(H_{0}\right)$, is just a first order perturbation approach with respect to the additional degrees of freedom which appear in the perturbation $\left(H_{1}\right)$. Moreover, the study of secondary resonances is made via the introduction of suitable averaged models, in each of which only one resonance is taken into account, neglecting the presence of all others.

These two facts make the method not very suitable to study the problems, or the regions of the phase space, characterized by a strong coupling between the degrees of freedom. In section 2 we have seen, for example, how the method underestimates the correct size of the chaotic layers. There are many problems of interest in Celestial Mechanics characterized by a strong interaction among degrees of freedom, which, for this reason, lay almost completely unexplored. We quote here the dynamics in mean-motion resonances at high inclination, the interaction among secular and mean-motion resonances, the motion of the Trojans. Therefore, it would be interesting to provide a practical extension of Henrard's method, to allow to go further from first order results. We stress here that this is not straightforward. Indeed the construction of suitable action-angle variables, which is the base of Henrard's method, can not be done analytically, but must be performed with numerical techniques; conversely, the application of the usual perturbation methods, such as the Lie's algorithm, requires explicit algebraic expressions. Therefore the extension of the method needs the developement of a new practical perturbation theory.

The new method we have developed is based on the successive eliminations of perturbation harmonics via the introduction of a sequence of suitable action-angle variable canonical transformations. The practical applicability of this method is largely illustrated in Morbidelli (1992), and the conditions under which this method can be considered as a genuine perturbation theory are studied in a pure theoretical way in Morbidelli and Giorgilli (1992). The aim of this section is just to illustrate the basic ideas, skipping all technical details.

\subsection{The algorithM}

The basic idea of our new approach is simple and dates from Delaunay. Consider, for example, a two degrees of freedom Hamiltonian $H\left(I_{1}, \phi_{1}, I_{2}, \phi_{2}\right)$. First we expand 
it in Fourier series with respect to the angles $\phi_{1}$ and $\phi_{2}$, so that we can write

$$
H\left(I_{1}, \phi_{1}, I_{2}, \phi_{2}\right)=H_{0,0}\left(I_{1}, I_{2}\right)+\sum_{n, m} h_{n, m}\left(I_{1}, I_{2}\right) \cos \left(n \phi_{1}+m \phi_{2}\right),
$$

where we have assumed, for simplicity, that only cos components are present in the expansion. Furthermore we choose one harmonic, with index $\bar{n}, \bar{m}$ say, and consider the partial Hamiltonian

$$
H_{0,0}\left(I_{1}, I_{2}\right)+h_{\bar{n}, \bar{m}}\left(I_{1}, I_{2}\right) \cos \left(\bar{n} \phi_{1}+\bar{m} \phi_{2}\right) .
$$

Since this Hamiltonian is integrable, new canonical action-angle variables are introduced in order to take it to be independent of the new angles.

If the phase portrait of (16) is not simple (i.e. it contains separatrices), the new variables can not be introduced all over the phase space, but different sets of variables must be introduced in the different topological regions of the phase space of (16) (regions of libration and circulation of the critical angle $\bar{n} \phi_{1}+\bar{m} \phi_{2}$ ), separated by singular surfaces. In this sense, however, one can still consider as global the transformation to angle-action variables, since it conserve the topological structure of the phase space of (16).

Furthermore, in each topological region, the remainder

$$
\sum_{n \neq \bar{n}, m \neq \bar{m}} h_{n, m}\left(I_{1}, I_{2}\right) \cos \left(n \phi_{1}+m \phi_{2}\right)
$$

is transformed in the new variables and expanded in Fourier series with respect to the new angles. In this way one gets a new Hamiltonian which has exactly the same form as (15). The advantage is that the relevant term $h_{\bar{n}, \bar{m}}\left(I_{1}, I_{2}\right) \cos \left(\bar{n} \phi_{1}+\bar{m} \phi_{2}\right)$ is no longer present, since its dynamical effect has been taken into account in the definition of the new variables. This allows to perceive the possibility of iterating the algorithm, in order to eliminate all the principal harmonics. In the following we will show in an evident way on an example how all this works in practice.

\subsection{AN APPLICATION OF THE METHOD OF SUCCESSIVE ELIMINATION OF HAR- MONICS TO THE MIRANDA-UMBRIEL PROBLEM}

We apply in this section the method of successive elimination of perturbation harmonics to the Hamiltonian (2), with a particular care to the region in between the resonance with argument $2 \sigma$ and the resonance with argument $\sigma-\nu$, which is characterized by a large chaotic layer.

When a single harmonic is taken into account as in (16), there exists a linear combination of the action variables that turns out to be a constant of motion. The dynamical effect of the considered harmonic can be analyzed on different surfaces corresponding to different values of this constant. Indeed, on each surface, the problem is essentially monodimensional.

The harmonic of the original problem (2) which has the strongest dynamical effect is the one with argument $2 \sigma$. In Figure 6 we show the phase space of (16), where $\bar{n}=2, \bar{m}=0$, on two surfaces associated to two different values of the constant of motion. The existence of three dynamical regions is evident. We concentrate 
$n=2 \quad m=0$
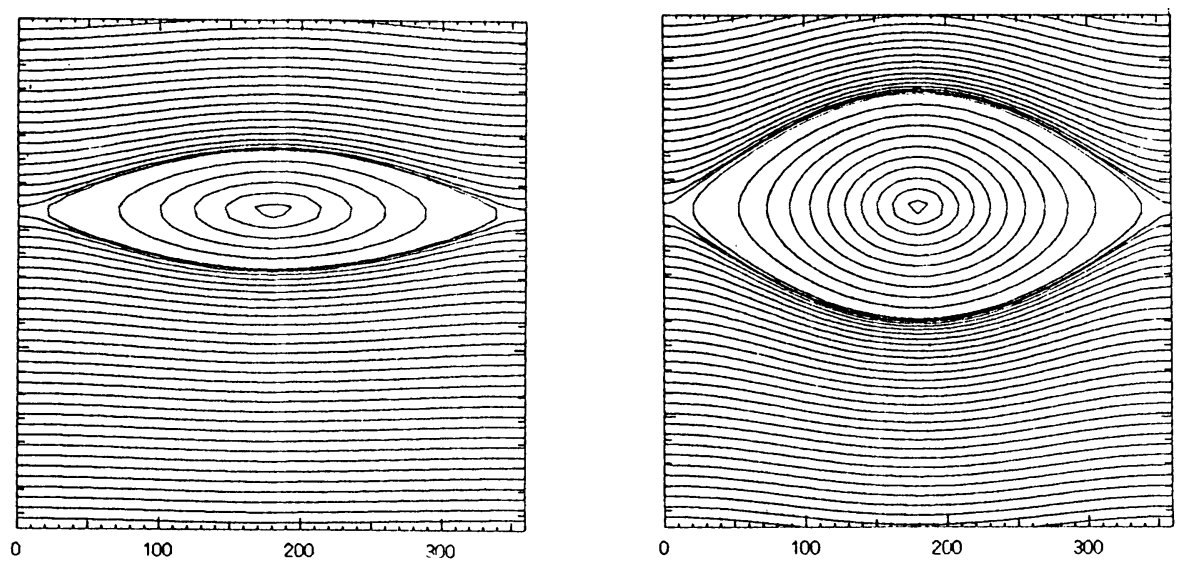

Fig. 6. The integrable dynamics obtained by considering only the perturbation harmonic with argument $2 \sigma$.
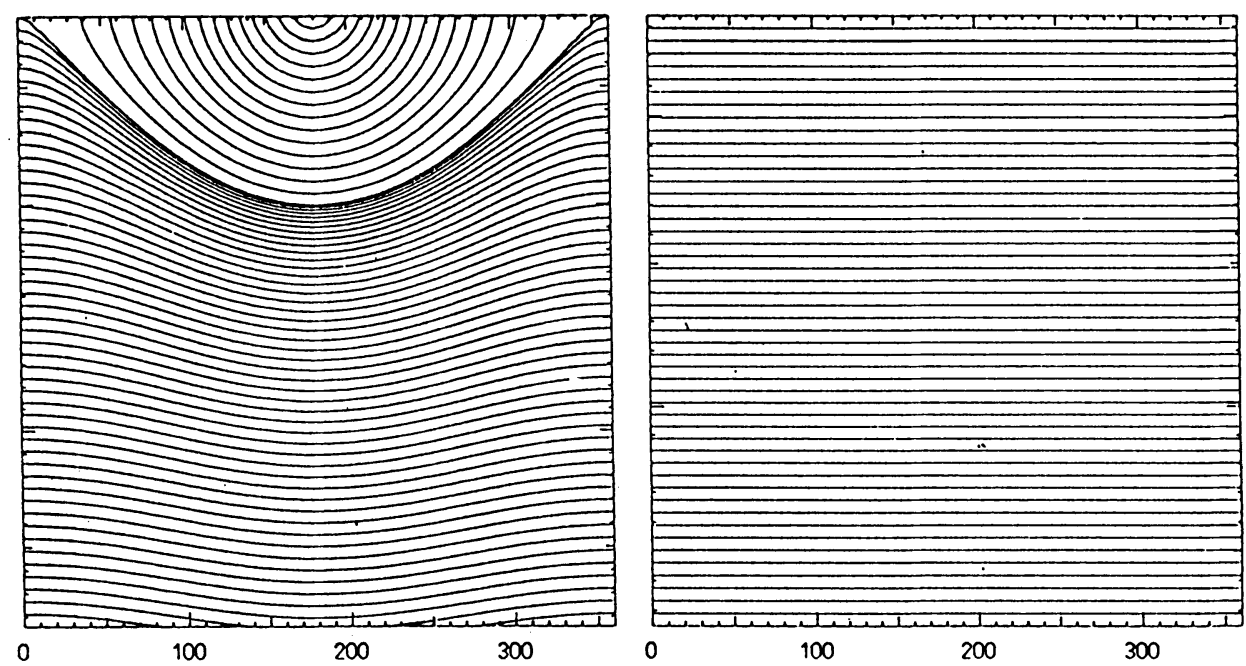

Fig. 7. The region of counterclockwise circulation of $2 \sigma$ in the original variables $2 \sigma, S$ (fig $a$, on the left) and in the new action-angle variables (fig b, on the right). 
$n=1 \quad m=-1$

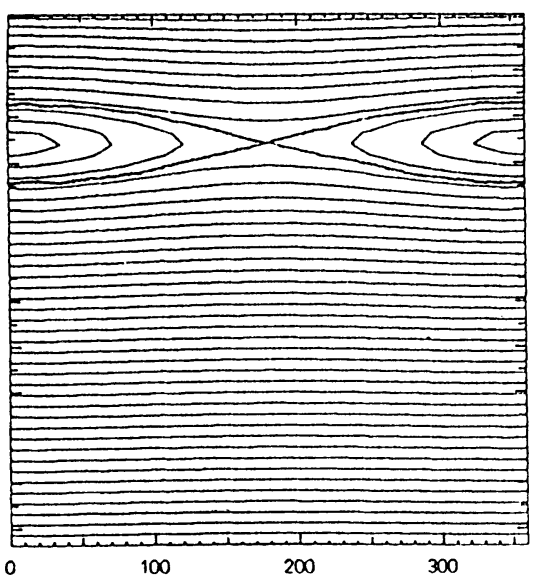

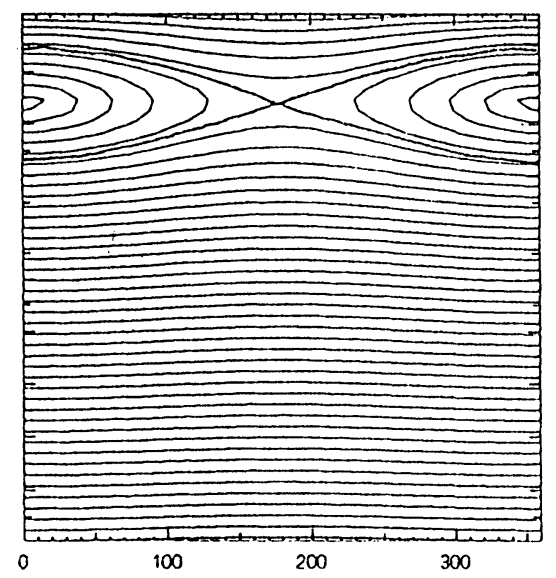

Fig. 8. The integrable dynamics obtained by considering only the perturbation harmonic with argument $\sigma-\nu$.

in the following on the region of counterclockwise circulation of the original angle $2 \sigma$, namely the region at the bottom of the pictures of Figure 6 . This region is magnified, for a given value of the constant of motion, in Figure 7a. From the topological point of view, the introduction of the new action-angle variables transforms the dynamical tori, which are distorted in the original variables, into hyperplanes which, on the considered surface, look as straight lines. For example, Figure $7 \mathrm{~b}$ is the same as Figure $7 \mathrm{a}$, but the dynamics is represented in the new action-angle variables. In particular, the separatrix, which is the bold line with extremes in the upper corners of Figure $7 \mathrm{a}$ in the old variables, is the upper border of Figure 7b in the new variables, and bounds the new domain of work. By looking at Figure $7 \mathrm{a}$ and Figure $7 \mathrm{~b}$, one easily understands why we speak of "elimination" of the harmonic; indeed in the new variables the dynamical effect of the harmonic has completely disappeared, since it is taken into account in the definition of the new variables themselves.

In the new domain of work, the harmonic with the most relevant dynamical effect turns out to be the one with argument $\sigma-\nu$ where, with an abuse of notation, we call as $\sigma$ and $\nu$ the new angles introduced for the elimination of the previously considered harmonic. In Figure 8 we show the phase space of the new integrable model, obtained by taking into account only the harmonic with argument $\sigma-\nu$, on two surfaces associated to two different values of the existent constant of motion. The orbits which collide with the upper border of the domain of work are lost, since one can not construct new action-angle variables on them. But, as the upper border is the image of the separatrix of the $2 \sigma$ resonance, we can expect that all these orbits lead to chaos, and in this way we can get an estimate of the size of the chaotic layer. In particular, if the separatrix of the $\sigma-\nu$ resonance collided against the upper border, we could foretell the existence of a bridge of chaos connecting 
$n=3 \quad m=-1$

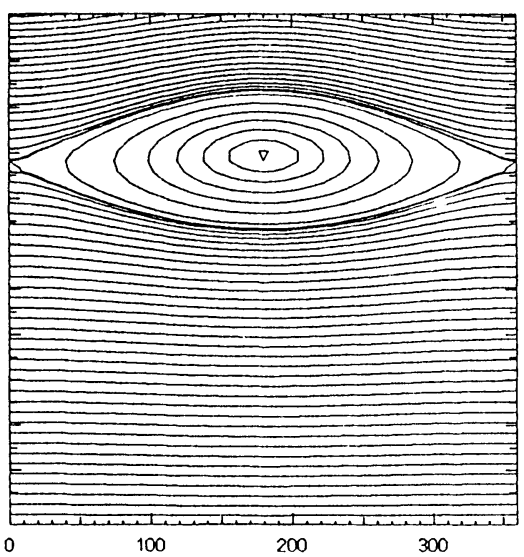

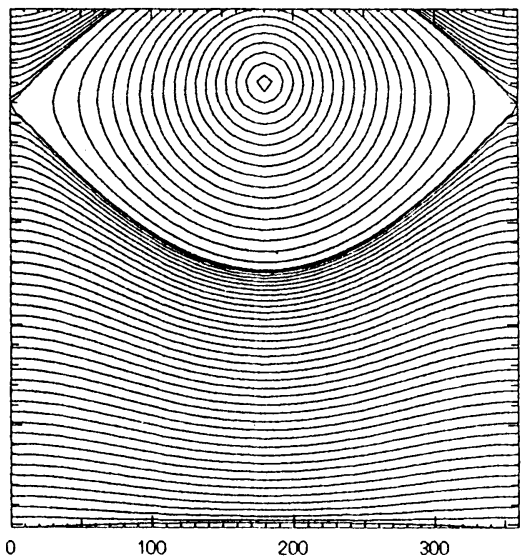

Fig. 9. The integrable dynamics obtained by considering only the perturbation harmonic with argument $3 \sigma-\nu$.

the $2 \sigma$ resonance and the $\sigma-\nu$ resonance. However, as one sees, this is never the case. This result has been already obtained in section 2; indeed, at this step, the results are completely equivalent to those obtainable by Henrard's method (1990). The advantage of the new method is that we are no longer compelled to stop at this step. We can introduce new action-angle variable in the region in between the two resonances, value the Hamiltonian in the new variables and expand it in Fourier series of the new angles, and look for the next harmonic to be eliminated.

The new most relevant harmonic turns out to be the one with argument $3 \sigma-\nu$, the dynamical effect of which is shown on two surfaces in Figure 9. We remark that this harmonic does not exist in the Hamiltonian (15) expressed in the original variables, and has been created by the introduction of the new variables. The upper border of each picture in Figure 9 is the separatrix of the $2 \sigma$ resonance, while the lower border is the separatrix of the $\sigma-\nu$ resonance. As one sees, in Figure $9 \mathrm{~b}$ the separatrix of the resonance collides against the upper border of the domain of work, so that we can foretell the existence of a bridge of chaos between the $2 \sigma$ resonance and the $3 \sigma-\nu$ resonance, at least on the surfaces above a critical one (i.e. for $i>0.06$ ). However a regular region seems to exist in between the $\sigma-\nu$ resonance and the $3 \sigma-\nu$ resonance. In that region we introduce new action-angle variables and look for the next harmonic to be eliminated. This turns out to be the one with argument $4 \sigma-2 \nu$, illustrated in Figure 10. New action-angle variables are furthermore introduced in the region between the $3 \sigma-\nu$ and the $4 \sigma-2 \nu$ resonances (on the surfaces where it exists), and in the region between the $4 \sigma-2 \nu$ and the $\sigma-\nu$ resonances. 
$n=4 \quad m=-2$

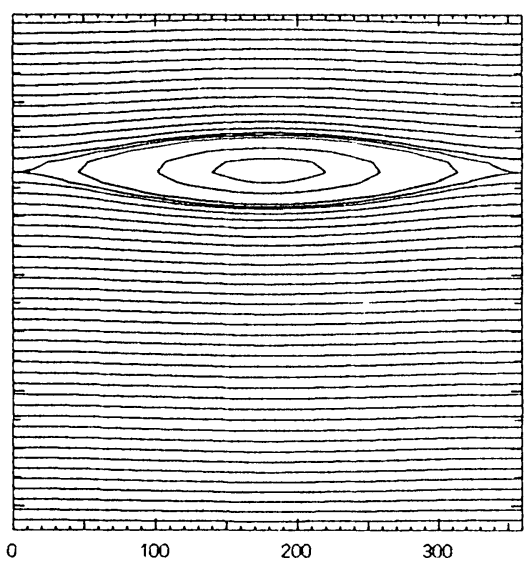

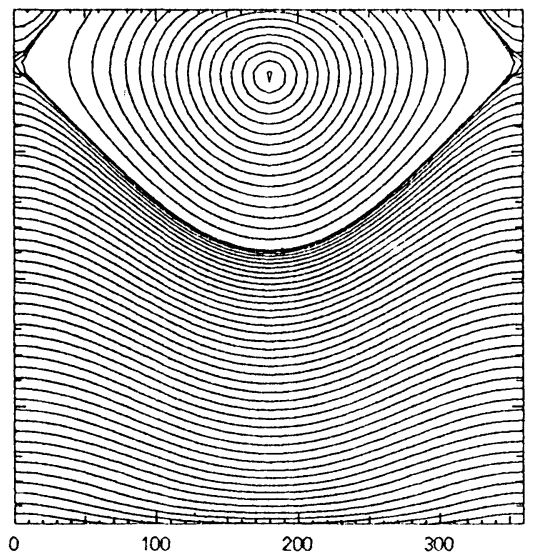

Fig. 10. The integrable dynamics obtained by considering only the perturbation harmonic with argument $4 \sigma-2 \nu$.

\subsection{The final determination of the size of the ChaOtic layers}

In the two regions where the new variables are defined, the Hamiltonian is no longer characterized by the presence of a "most relevant" harmonic, but by the existence of many resonant harmonics, all with approximately the same width. In principle, one could try to eliminate them, one after the other. However, this turns out to be impractical, from the point of view of computing time and accumulation of numerical errors. What we decide to do, at this step, is to plot in an action-action diagram (that corresponds approximately to a $a, i$ diagram) the width of all of them and to see in which region the resonances overlap. This is done in Figure 11a for the region in between the $4 \sigma-2 \nu$ and the $\sigma-\nu$ resonances, and in Figure 11b for the region in between the $3 \sigma-\nu$ and the $4 \sigma-2 \nu$ resonances. Applying now Chirikov criterion, we estimate the size of chaotic layers as the size of the regions where overlapping of resonances occurs. The four dashed lines plotted in Figure 11a denote the energy levels where Moons and Henrard have computed by numerical integration the Poincaré sections that we report in Figure 12. On each picture of Figure 12 the coordinates used are the semi major axis $a$ on the $x$ axis (ranging from 0.4806 to 0.4809$)$ and $i \cos (\sigma+\nu)$ on the $y$ axis (ranging from -0.1 to +0.1 ). The Poincaré sections are made at $2 \sigma=\pi$. On the pictures we report the critical arguments corresponding to each secondary resonance. The big region in between the two large chaotic layers, is the region of libration of the argument $2 \sigma$. Let us concentrate on the region in between the $\sigma-\nu$ resonance and the $2 \sigma$ resonance. As one sees, in the first two Poincaré sections (corresponding to levels 1 and 2 in Figure 11a) a bridge of chaos connects the two resonances. This is in agreement with the results of Figure 11a, since the corresponding levels are in the region of resonance overlapping. On the contrary, in the third and fourth Poincaré sections, the $\sigma-\nu$ resonance is detached from the chaotic layer. Indeed the corresponding 

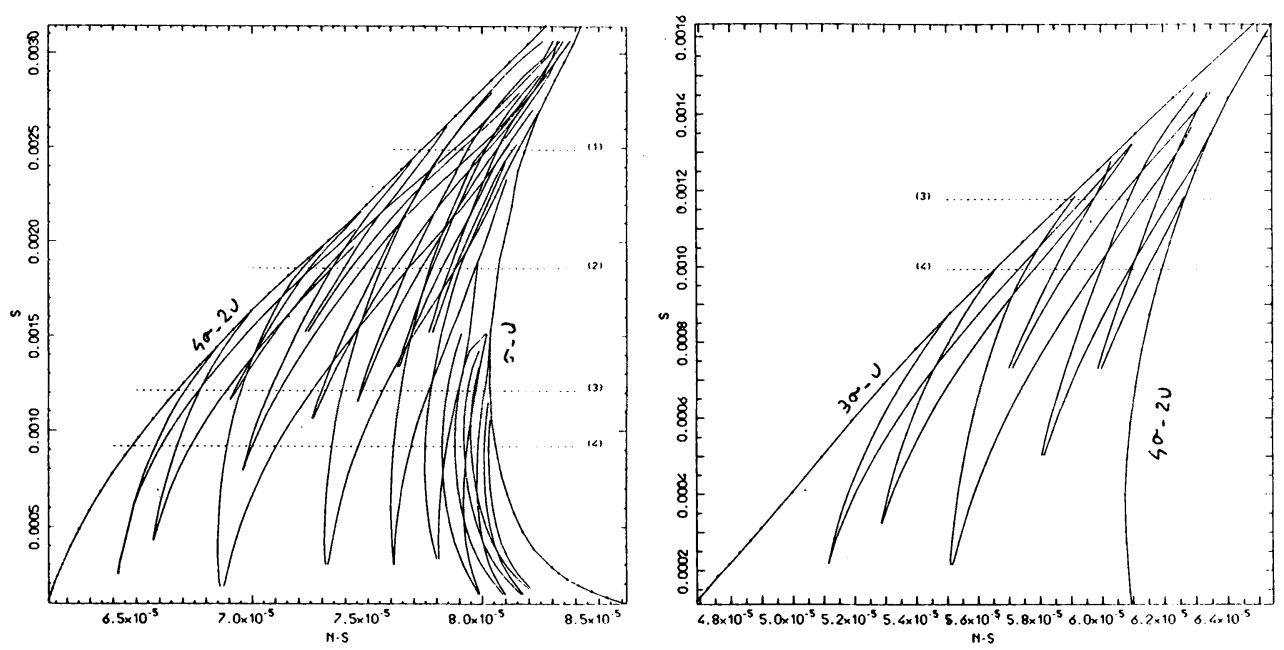

Fig. 11. The overlap of secondary resonances in the region in between the $4 \sigma-2 \nu$ and the $\sigma-\nu$ resonances (fig. a on the left), and in the region between the $3 \sigma-\nu$ and the $4 \sigma-2 \nu$ resonances

levels 3 and 4 in Figure 11a lay in the region where the overlapping of resonances does not occur. Moreover in Figure 12 we recognize the islands associated with the $4 \sigma-2 \nu$ and the $3 \sigma-\nu$ resonances, thus confirming the fine structure of secondary resonances we have detected in section 4.2. The smaller resonances in between the $4 \sigma-2 \nu$ and $\sigma-\nu$ commensurabilities are too faint to be detected in Figure 12 where they do not give origin to the chaotic layer.

For what regards the region between the $3 \sigma-\nu$ and the $4 \sigma-2 \nu$ resonances, we remark in Figure 12 that, on the third section, the chaotic layer reaches the $4 \sigma-2 \nu$ resonance, while, on the fourth section, it stops at a very small island corresponding to the resonance with argument $7 \sigma-3 \nu$. In Figure $11 \mathrm{~b}$ we see that level 3 , that corresponds to the third section in Figure 12, crosses the region where resonances overlap; conversely level 4 is at the limit of the region of resonant overlapping. Moreover the widest resonance at the center of Figure $11 \mathrm{~b}$ is the one with argument $7 \sigma-3 \nu$.

Such a precision in the determination of the size of the chaotic layers may be astonishing. Indeed it is well known that Chirikov criterion in general provides just rough estimates, since it does not take into account the mutual interaction among the resonances. However we remark that we apply here Chirikov criterion to an Hamiltonian that has been treated by the perturbation method of elimination of harmonics up to a pretty high order, so that to achieve the description of the fine structure of secondary resonances. Chaos is determined at this stade by the overlapping of many faint resonances together, so that it is intuitive that the neglected 


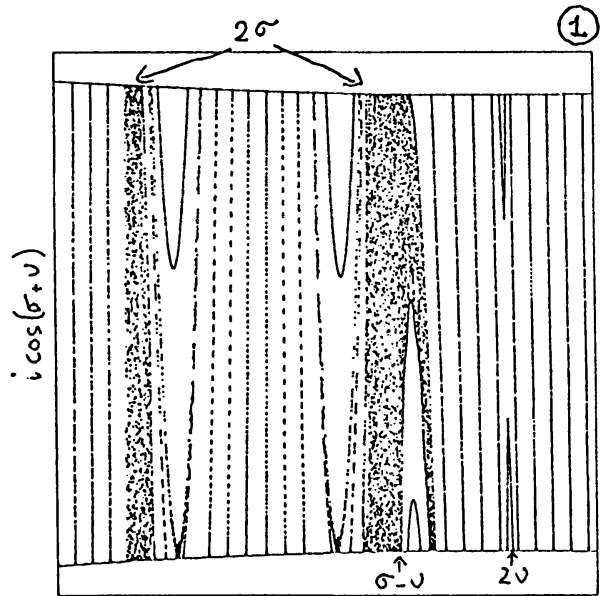

$a$

(3)

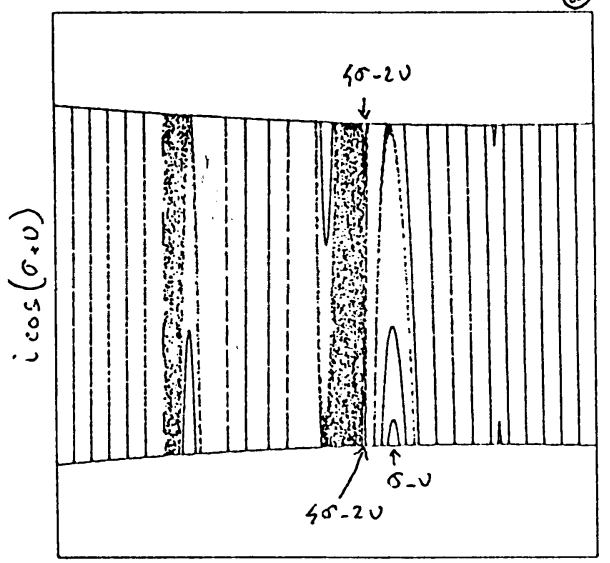

a

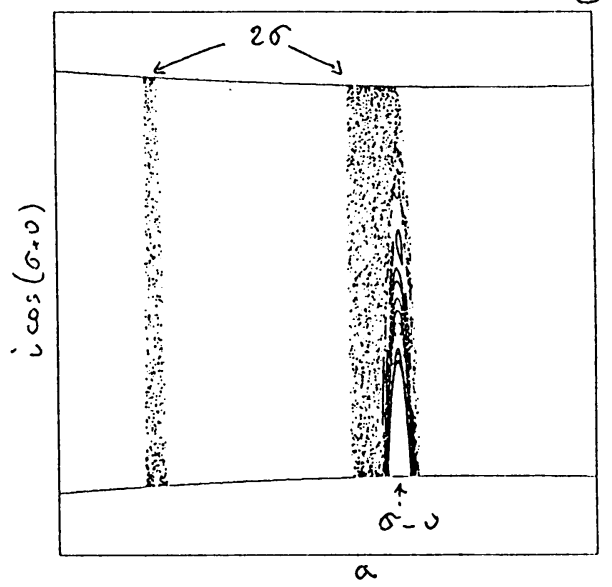

(4)

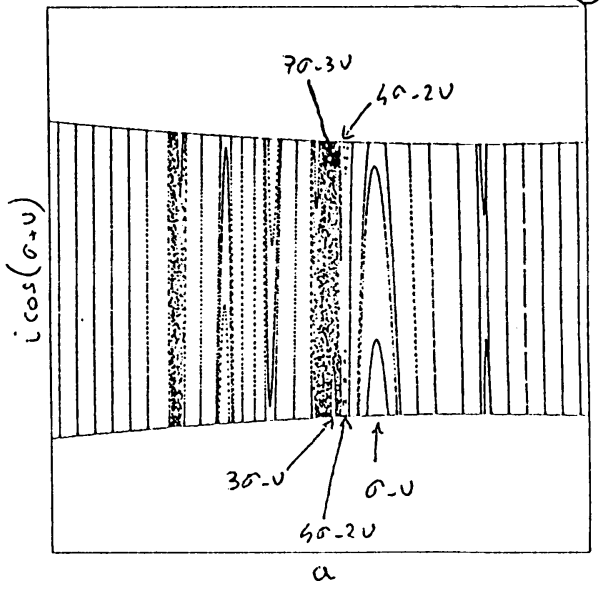

Fig. 12. Poincaré sections obtained by numerical integration. 
mutual interactions can not change the picture as much as they do when just $t-$ wo strong resonances are taken into account, for example working on the original Hamiltonian (15). In particular a well defined threshold of transition appear to exist between the region where resonances do not overlap and the region where they overlap. The location of this threshold seems to be very unsensitive to the numerical accuracy of the computations.

\subsection{Theoretical RESULTS ON THE SUCCESSIVE ELIMINATIONS OF HaRMonics}

In the paper by Morbidelli and Giorgilli (1992), the method of successive eliminations of harmonics is analyzed from the pure theoretical point of view. In particular it is shown that, under suitable assumptions, the method can be considered as a genuine perturbation theory. Indeed, the authors prove that, if some condition of smallness of the original perturbation is satisfied, one could in principle perform an arbitrary number of steps of the algorithm of successive elimination of harmonics. And if this number is pushed to infinity, then the algorithm converges on the so called KAM-tori. This, of course, can not be done in practice. What one can do in practice, on the contrary, is to perform a finite number of steps. The meaning of this, from the theoretical point of view, is that one constructs a (open) set of quasi-invariant tori. The action variables which parameterize these tori are not constants of motion, but have a "slow" diffusion (approximately exponentially small with the order of the non yet eliminated harmonics). And the further one goes in the application of the algorithm, the better are the action variables one constructs.

From the point of view of the theory of general dynamical systems, this can be read as a Nekhoroshev like result in the neighbourhood of the KAM-tori. Indeed, by the successive elimination of harmonics, one can prove that Arnold diffusion is exponentially slow with the inverse of the distance of the starting point from the closest KAM-torus.

\section{References}

Arnold, V., I., (1963): "On a theorem of Liouville concerning integrable problems of dynamics", Sib. mathem. zh., 4, 2.

Delaunay, C., (1867): "Theorie du mouvement de la Lune", Mem. Acad. Sci., Paris, 29

Dermott, S.F.: 1984, "Origin and evolution of the Uranian and Neptunian satellites: some dynamical considerations", in Uranus and Neptune (J Bergstrahl Ed.), Nasa Conf. Pub. 2330, pp 377-404.

Dermott, S.F., Malhotra, R. and Murray C.D.: 1988, "Dynamics of the Uranian and Saturnian satellite systems : A chaotic route to melting Miranda ?".Icarus,76, 295-334.

Goldreich, P.: 1965, "An explanation of the frequent occurence of commensurable mean motions in the Solar system", M.N.R.A.S., 130, 159-181.

Henrard, J.: 1982, "Capture into resonance : An extension of the use of the adiabatic invariants", Celest. Mech., 27, 3-22.

Henrard, J.: 1990, "A semi-numerical perturbation method for separable Hamiltonian systems", Celest. Mech., 49, 43-68.

Henrard, J. and Lemaître, A.: 1983, "A second fundamental model for resonance",Celest. Mech., 30, 197-218. 
Henrard, J., and Lemaître, A.: 1986, "A perturbation method for problems with two critical arguments", Celest. Mech., 39, 213-238.

Henrard, J., and Moons, M.: 1992, “Capture probabilities for secondary resonances", Submitted to Icarus.

Malhotra, R. and Dermott, S.F.: 1990, "The role of secondary resonances in the orbital history of Miranda", Icarus, 85,444-480.

Moons, M., and Henrard, J.: 1992, "Surfaces of section in the Miranda-Umbriel 1:3 inclination problem", Submitted to Celestial Mechanics.

Morbidelli, A. (1992): "On the successive eliminations of perturbation harmonics", submitted to Celest. Mech.

Morbidelli, A., and Giorgilli, A., (1992): "Quantitative perturbation theory by successive elimination of harmonics", in preparation.

Peale, S.J.: 1986, "Orbital resonance, unusual configurations and exotic rotation states", in Satellites (J. Burns and M. Matthews eds.), Univ. of Arizona Press, 159-223.

Peale, S.J.: 1988, "Speculative histories of the Uranian satellite system", Icarus, 74, 153171.

Tittemore, W.C. and Wisdom, J.: 1988, "Tidal evolution of the Uranian satellites. I. Passage of Ariel and Umbriel through the 5:3 mean-motion commensurability", Icarus, 74, 172-230.

Tittemore, W.C. and Wisdom, J.: 1989, "Tidal evolution of the Uranian satellites. II. An explanation of the anomalously high orbital inclination of Miranda",Icarus, 78, 63-89.

Tittemore, W.C. and Wisdom, J.: 1990, "Tidal evolution of the Uranian satellites. III. Evolution through the Miranda-Umbriel 3:1, Miranda-Ariel 5:3, and Arial-Umbriel 2:1 Mean-Motion Commensurabilities", Icarus, 85, 394-443.

Yoder, C.: 1973, "On the establishment and evolution of orbit-orbit resonances", Ph.D. Dissertation, U. of California, Santa Barbara.

Yoder, C.F.: 1979, "Diagrammatic theory of transition of pendulum-like systems", Celest. Mech., 19, 3-29. 


\section{Discussion}

R.A.Broucke (to J.Henrard) - Can you make some comments about the importance of the role played by $J_{2}$ (the oblateness of the central body) in your work?

J.Henrard - The role of $J_{2}$ is to separate the unperturbed frequency of the various periodic terms. The interaction of the various periodic terms will depend upon its value.

J.Laskar (toJ.Henrard) - Can you explain further, why when trapped in the primary resonance and when the eccentricity increases, the satellite is not trapped in the $4 / 1$ or $3 / 1$ secondary resonances, but only in the $2 / 1$ resonance?

J.Henrard - There is a probability of capture associated with each secondary resonance. Low probability for secondary resonances $N / 1$ with large $N$ and probability equal to one for the $2 / 1$ secondary resonance. The particular simulation $I$ have shown escape from all the $N / 1$ (with $N>2$ ) secondary resonances and ends up in the $2 / 1$ secondary resonance, but other cases are posssible.

J.Laskar (to A.Morbidelli) - In your iterative process, you start from a given resonance. When you define the second coordinates transformation, it should change the location of the first resonance. In other words, what happens if you start from a different resonance in your process?

A.Morbidelli - It is true that, once a resonance has been eliminated, its location cannot be changed by the elimination of the successive harmonics. However, the elimination of a resonant harmonic defines the borders of the domain of work and, when new variables are defined in order to eliminate a further harmonic, a portion of volume, given by the tori that collide with the borders, must be eliminated. We believe that this volume is lost in the chaotic zone. The effect of the second harmonic on the first one is, then, the creation of a chaotic zone around the separatrices.

When a hierarchy clearly exists among resonances, the order by which the harmonics must be eliminated is evident. When two resonances have the same strength, the order of elimination is arbitrary. Some tests we have performed changing the sequence of elimination have given the same results. From a pure theoretical point of view, one can show that the intersection of the regular domains obtained by eliminating the harmonics in different sequences, is non-empty. 